\title{
Achieving TASAR Operational Readiness
}

\author{
David J. Wing* \\ NASA Langley Research Center, Hampton, VA 23681, USA
}

\begin{abstract}
NASA has been developing and testing the Traffic Aware Strategic Aircrew Requests (TASAR) concept for aircraft operations featuring a NASA-developed cockpit automation tool, the Traffic Aware Planner (TAP), which computes traffic/hazard-compatible route changes to improve flight efficiency. The TAP technology is anticipated to save fuel and flight time and thereby provide immediate and pervasive benefits to the aircraft operator, as well as improving flight schedule compliance, passenger comfort, and pilot and controller workload. Previous work has indicated the potential for significant benefits for TASAR-equipped aircraft, and a flight trial of the TAP software application in the National Airspace System has demonstrated its technical viability. This paper reviews previous and ongoing activities to prepare TASAR for operational use.
\end{abstract}

\section{Introduction}

A $\mathrm{N}$ opportunity is emerging for aircraft operators to significantly improve their flight operations by using advanced cockpit automation designed to optimize flight trajectories in real time. This opportunity is supported by the emergence of three systems: the Electronic Flight Bag (EFB), Automatic Dependent Surveillance Broadcast (ADSB) IN, and broadband internet connectivity in the cockpit. The EFB provides a platform for powerful applications to have direct access to real-time flight data from onboard avionics. Therefore, as the flight proceeds and conditions evolve, the connected EFB applications can act immediately with the most accurate and up-to-date information about the flight. ADS-B IN provides the ability for the cockpit automation to receive the same high-quality traffic surveillance information viewed by Air Traffic Control (ATC), thereby providing an unprecedented awareness of the local traffic environment that can be used to increase the frequency of user-request approvals by ATC. Finally, airborne internet provides networked connectivity to an array of external information on operational factors such as wind predictions, weather hazards, and Special Activity Airspace (SAA) status, giving the automation a real-time status of the aircraft's operating environment. The integration of these systems and data sources with advanced cockpit automation yields a powerful combination that empowers aircraft operators to take a much more proactive role in managing the flight than is otherwise possible today. Armed with this advanced trajectory-replanning capability, aircrews can more effectively and efficiently work with air traffic controllers by making flight-optimizing trajectorychange requests that address some of the controller's primary concerns, enabling them to more often approve such requests. The result is a win-win for users and controllers: users will more often receive their desired trajectory improvements (whatever they may be) and controllers will save workload through having fewer problematic or nonapprovable requests. In addition, for users that have a dispatch service, the cockpit automation can aid coordination between the dispatchers and pilots to ensure fleet requirements are met and consistent strategies are used to optimize the post-departure flight.

Since 2012, NASA has been developing and testing the Traffic Aware Strategic Aircrew Requests (TASAR) concept $^{1,2,3}$ for aircraft operations featuring a NASA-developed cockpit automation tool, the Traffic Aware Planner (TAP) ${ }^{4}$ which computes traffic/hazard-compatible route changes to improve flight efficiency. The technology is anticipated to save fuel and flight time and thereby provide immediate and pervasive benefits to the aircraft operator, as well as improving flight schedule compliance, passenger comfort, and pilot and controller workload. TASAR represents a seminal advancement of the aircraft's role in airspace operations, promoting it from just a passive recipient of ATC instructions to an active trajectory manager in the dynamic airspace. NASA's Aeronautics Research Mission Directorate is strategically focused on promoting operational autonomy in the Next Generation Air Transportation System (NextGen) and beyond, ${ }^{5}$ and TASAR is expected to be a principal catalyst towards this transformation.

\footnotetext{
* ATM Research Engineer, Crew Systems \& Aviation Operations, Mail Stop 152, david.wing @ nasa.gov, AIAA member
} 
The paper will provide a status on TASAR research and development as it has rapidly progressed from concept formulation to operational-use readiness in the National Airspace System (NAS). Section II provides acknowledgements. Section III will discuss the TASAR project approach. Section IV will review previously reported accomplishments since project inception, culminating with the initial flight testing of the TAP software in November 2013. Section V will address current activities in preparation for upcoming operational-use trials with NASA's TASAR-partner airlines. Section VI will discuss future directions, and Section VII will present conclusions.

\section{Acknowledgements}

Much of the work reported in this paper has been accomplished under NASA contracts by the following organizations operating as a cohesive TASAR team with the author and his NASA colleague, Dr. Kelly Burke, the project's human-factors lead. Engility Corporation, supported by Stinger Ghaffarian Technologies, designed and developed the TAP software, conducted analyses of benefits and data sources, and performed extensive engineering analysis and design for aircraft adaptation of the TAP software. Advanced Aerospace Solutions (AdvAero) conducted the TASAR flight trials on their Piaggio Avanti P.180 flight-test aircraft. Rockwell Collins performed analyses of TASAR certification and operational approval requirements, including assessing safety / operation hazards and regulatory policies for TASAR as an EFB application. The University of Iowa's Operator Performance Lab conducted human-factors evaluations in two simulation experiments. Cotton Aviation Enterprises through the National Institute of Aerospace provided extensive operational expertise, guidance, and design. MYMIC contributed to the TAP humanmachine interface design and the development of a computer-based trainer.

\section{Approach}

The goal of the TASAR project is to develop a near-term application of networked cockpit automation technology that can be readily adopted by a wide variety of airspace users for immediate operational benefit and that provides the foundational capability for more advanced applications leading towards greater operational autonomy in the future, such as described in [6]. NASA's approach for achieving this goal has been to leverage its research-prototype automation technology for airborne trajectory management, the Autonomous Operations Planner, ${ }^{7}$ previously developed and matured over a dozen years for advanced self-separation applications, and apply it towards a conservative, low-cost application that can be used by operators in the current NAS to optimize their flight. To rapidly make progress toward this goal, the project strategy shown in Figure 1 was employed.

1. Define a viable near-term, low-cost, operational concept, and assess its benefits $\mathrm{w} /$ users

\section{Assess FAA certification} and operational approval requirements, and vet with $\mathrm{FAA}$
3. Develop the technology and mature it through simulations and flight trials
4. Partner with airlines for operational-use testing, leading to tech transfer
5. Leverage operational

usage to mature \& expand

to future applications

Figure 1 TASAR project strategy.

The first step in the strategy is to define and document a viable operational concept for this technology that is fully compatible with current-day operational procedures and requires no policy changes or new technology anywhere other than onboard the aircraft. Assessing the potential user benefits is an important element of the strategy at this early stage, as is vetting the concept and potential benefits with potential users through an outreach effort to gauge user interest. The second step in the strategy is to ensure existing Federal Aviation Administration (FAA) regulatory documents would permit certification and operational use of such an application installed on an EFB, and then to follow up through direct engagement with FAA policy makers to confirm acceptability. The third step in the strategy is to produce the actual software application to be used by operators in the NAS, with the intent to make the software available through licensing directly from NASA or through third-party commercial vendors. This step in the strategy includes reducing risk through several significant activities in simulations, flight tests, and analyses to mature the software and its interfaces (i.e., data and human user), to assess the human factors issues associated with adding this application to current cockpit procedures, and to ensure its readiness for use in an operational environment. Fourth in 
the strategy is to seek airline partners to conduct operational-use testing on revenue flights, during which any residual real-world operational issues would be identified and resolved. At this point, the technology would be ready for adoption by the wider user community, while NASA would move to the fifth step in the strategy, which is to work with users and industry to mature and expand the technology's use to future applications. Such applications would likely leverage additional air-ground integration while pursuing a path towards greater operational autonomy for aircraft in future operations.

\section{Completed Activities: Concept \& Technology Development}

The TASAR project was started in 2012 under the sponsorship of the NASA Airspace Systems Program through the NextGen Concepts \& Technology Development Project, and it continues under the NASA Airspace Operations and Safety Program through the Airspace Technology Demonstration Project. The TASAR project set an aggressive schedule of developing the concept and technology and achieving initial flight testing within two years. A number of significant activities contributed to this achievement (accomplished in just 20 months), most of which were previously reported in AIAA conferences and other venues. These activities will be summarized in the sub-sections below.

\section{A. Concept Development}

The TASAR concept combines ADS-B surveillance and flight deck automation for in-flight decision-aiding and replanning to increase the likelihood of ATC approval of pilots' trajectory change requests, thereby improving the efficiency or other user-desired attribute of the flight, while not adversely impacting and potentially benefiting the air traffic controller. In addition to ADS-B surveillance, TASAR can leverage ground-based information services via data link or internet access, as well as on-board weather radar, to identify weather hazards to be avoided and other conditions affecting flight optimization and ATC approval. The TASAR operational concept was presented in 2012, ${ }^{1}$ a Concept of Operations document was published in $2013,{ }^{2}$ and a patent was issued in $2015 .^{3}$ An analysis of how TASAR achieves the goals of low-cost implementation and near-term applicability was presented in $2013 .{ }^{8}$

The TASAR concept was developed with several key attributes, shown in Table 1, the combination of which make it unique among flight-optimization concepts and technologies. As a "per-aircraft" capability, TASAR can be implemented by early adopters at a cost-beneficial deployment rate determined by the user and without dependence on other aircraft or users to be TASAR equipped to start realizing a return on investment. Important to its viability for rapid adoption, TASAR can be implemented with no changes to FAA systems and polices, no changes to pilot/controller responsibilities, and no special consideration (i.e., operational credit) given to TASAR equipped aircraft. TASAR enables a more consistent accrual of user benefits by leveraging the aircrew's attention and low workload during en-route flight to pursue flight optimization opportunities that otherwise might be lost due to periods of high dispatcher workload. Finally, TASAR has the hallmarks of a good investment: low up-front costs and opportunities for future functional enhancement, integration, and expansion as the business case dictates. These opportunities include, for example, the potential to integrate TASAR capability directly into the avionics, to link with dispatch operations for improved coordination, to connect to more external and onboard data sources for increased effectiveness, and to integrate with data communications when available allowing more complex requests to be exchanged with ATC. Together, these attributes provide users with a solid business-case framework to implement this flight-optimization capability with low risk in current-day operations.

Table 1 Key attributes of the TASAR concept.

\begin{tabular}{|l|l|}
\hline Attribute & Benefit \\
\hline Per-aircraft capability & Early adopter benefit \\
\hline No changes to existing FAA systems \& policies & Implementation benefit \\
\hline No changes to aircrew / ATC roles and responsibilities & Procedures / training benefit \\
\hline No operational credit requested for TASAR users & Operational approval benefit \\
\hline Leverages aircrew availability / low workload en route & Opportunity benefit \\
\hline Non-safety-critical intended function & Certification cost benefit \\
\hline Compatible with low-cost EFB hardware & Equipage cost benefit \\
\hline Ripe for future integration and functional growth & Investment benefit \\
\hline
\end{tabular}

\section{B. Preliminary Benefits Assessment}

A preliminary benefits assessment was conducted at the beginning of the TASAR project and was published and presented in 2012. ${ }^{9}$ A fast-time simulation model using an application programing interface to the Future Air-Traffic- 
Management Concept Evaluation Tool (FACET) ${ }^{10}$ was implemented and used to analyze historically flown trajectories in comparison to optimized trajectories using a TASAR capability for making trajectory-change requests to ATC. The platform included models for airborne surveillance, conflict probing, flight optimization, aircrew requests, ground surveillance, and ATC evaluation/response. Allowable trajectory changes included lateral-only, vertical-only, and combination lateral/vertical changes to optimize a specified parameter: fuel burn, flight time, or a weighted combination. Three TASAR use cases were modeled: recovery from an expired reroute initiative, weatherimpacted rerouting, and wind-optimal rerouting. Aircraft types and stage lengths representing a variety of user classes - network carriers, low-cost carriers, regional airlines, and business aviation - were modeled. A total of 510 flights from July 11 to July 20, 2012, were analyzed, and 300-2000 alternative TASAR trajectories for each flight were evaluated at five minute intervals. Further details of the analysis methodology and assumptions can be found in [9]. The airport pairs analyzed and the estimated benefits per operation are shown in Figure 2.

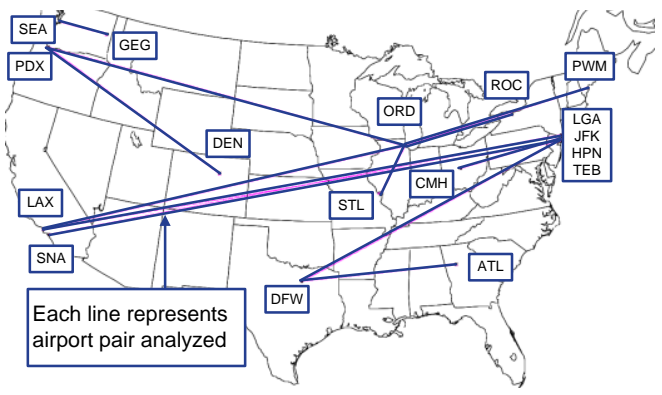

\begin{tabular}{|c|c|c|c|c|c|c|}
\hline \multirow{2}{*}{$\begin{array}{c}\text { Class of } \\
\text { Airspace } \\
\text { User }\end{array}$} & \multicolumn{2}{|c|}{$\begin{array}{c}\text { Time } \\
\text { Objective }\end{array}$} & \multicolumn{2}{c|}{$\begin{array}{c}\text { Fuel } \\
\text { Objective }\end{array}$} & \multicolumn{2}{c|}{$\begin{array}{c}\text { Time/Fuel } \\
\mathbf{5 0 / 5 0} \text { Obj. }\end{array}$} \\
\cline { 2 - 7 } & TS & FS & TS & FS & TS & FS \\
\hline Network & $\mathbf{4 . 2}$ & -122 & 3.4 & $\mathbf{5 7 5}$ & 3.6 & 543 \\
\hline Low Cost & $\mathbf{2 . 9}$ & -123 & 2.5 & $\mathbf{4 0 6}$ & 2.6 & 344 \\
\hline Regional & $\mathbf{1 . 0}$ & -88 & 0.8 & $\mathbf{1 3 7}$ & 1.0 & 66 \\
\hline Business & $\mathbf{1 . 2}$ & -22 & 1.6 & $\mathbf{6 4}$ & 1.5 & 53 \\
\hline
\end{tabular}

Figure 2 Preliminary benefits analysis results. TS: mean time savings (minutes) per operation. FS: mean fuel savings/cost (pounds) per operation.

Results from this preliminary analysis indicated that, in general, benefits increased with longer stage lengths since beneficial trajectory changes can be applied over a longer distance. Also, larger benefits were experienced between large hub airports as compared to other airport sizes. This was largely due to less efficient (from an airspace user point of view) historically flown trajectories between large hub airports and not all flown trajectories operating to or from medium hub or smaller airports having significant room for improvement. On average, aircraft equipped with TASAR, relative to aircraft not equipped with TASAR, saved about one to four minutes of time per operation and about 50 to $550 \mathrm{lbs}$ of fuel per operation, depending on the objective of the aircrew (time, fuel, or weighted combination of time and fuel), class of airspace user, and aircraft type.

\section{Safety and Operational Hazards Assessment}

An assessment of TASAR safety and operational hazards was conducted by Rockwell Collins and presented in $2013{ }^{8,11,12,13}$ Two safety assessment methodologies compliant with the FAA's Safety Management System were used to determine the Failure Effects Classification (FEC) of TASAR. Method 1, based on ARP 4761 [14], AC 23-1309 [15] and AC 25-1309 [16] for Part 23 and Part 25 aircraft operations, is a traditional system safety process for airborne systems, e.g., TASAR. Using the intended function of the new system capability, it identifies failure events, examines their effect on aircraft, aircrew, and ATC, determines a hazard classification and frequency of occurrence, and provides a rationale for the assessment. Method 2, based on RTCA DO-264 / EUROCAE ED 178A Operational Safety Analysis [17], represents a system-of-systems analysis approach well-suited for allocating safety requirements across a multiple-system function, normally reserved for higher criticality systems than TASAR. It involves identifying Operational Effects, Severity Classes, Effect Probabilities, Safety Objectives, Abnormal Events, Basic Causes, and External/Internal Mitigation Means. This second method was not performed in totality but was used to confirm the findings of the first method.

The two safety analyses concluded that the worst case FEC for TASAR would likely be determined to be "No Effect" and no higher than "Minor Effect." This preliminary determination is subject to evaluation and approval by cognizant FAA certification and operational approval organizations responsible for authorization of EFB applications. Supporting detailed rationale for the "No Effect" designation is provided in [11].

Several key factors influenced the FEC determination for TASAR. TASAR is a supplemental and advisory system and thus not relied on by critical functions supporting flight deck operations. Its use is optional, i.e., not a required system for flight operations. In the event of failures of the TASAR system, it can be ignored or disabled without adversely affecting operations. TASAR has no Minimum Equipment List requirement, and its presence or loss does not change responsibilities of the aircrew for flight operations. Change request procedures are unchanged. The aircrew 
must direct all change requests to ATC using conventional means, and ATC remains responsible for reviewing request for acceptability, including separation from traffic and restricted airspace. Undetected, misleading information associated with TASAR recommendations will have "No Effect" or at most "Minor Effect" on the pilot, aircraft, or ATC. Whether due to failure of one of the TASAR sub-systems and associated automation processing, or being the result of inaccurate data obtained from ground-based or flight deck systems, spurious change requests are mitigated by flight crew inspection of the recommended trajectory change with cross-reference to onboard certified systems and by mitigations associated with the existing ATC process for change requests received from pilots.

NASA and Rockwell Collins met with FAA certification (AIR) and operational approval (AFS) authorities and presented the approval requirements analysis described in [11] to them. The FAA officials indicated that TASAR would warrant a "Minor Effect" FEC due to workload considerations, but noted that from an operational failure perspective, the "No Effect" designation is justified.

\section{Certification and Operational Approval Requirements Analysis}

In addition to assessing safety and operational hazards, Rockwell Collins assessed TASAR requirements for adherence to EFB standards, as well as FAA guidelines and regulatory requirements for TASAR operational approval. The analysis was documented and presented in $2013^{12}$ and further documented in $2015 .{ }^{13}$ These references identify 17 regulations, standards, and guideline documents that were reviewed for this analysis, many of which relate to the design and installation of EFB hardware and associated software applications. As a new EFB application, TASAR does not map directly into previously defined Type A or Type B applications. Though it has many of the characteristics of a Type B application, it is somewhat less stringent than typical Type B applications (e.g., those that compute aircraft performance values such as landing runway length) in terms of FEC per its intended function. In the NASA/Rockwell Collins meeting with the FAA, the AIR and AFS authorities confirmed the TASAR application will be considered Type B, despite it not be explicitly listed in AC 120-76. ${ }^{18}$ Type B software applications do not require compliance with DO-178. ${ }^{19}$

TASAR is designed to be implemented on a Class 2 Portable Electronic Device or Portable EFB. The Class 2 EFB requirement is due to the read-only interface needed by TASAR to access on-board avionics systems and data link connectivity via installed antennas for accessible external information sources, e.g., weather information. The EFB standards adherence requirements examined are expansive and detailed in nature. Reference [13] documents these results, specifically the roles and responsibilities of the operator, manufacturer, installer, avionics vender, and also of FAA certification and operational approvers of newly developed EFB applications, such as TASAR.

Anticipating a possible need for operators to pursue a formal certification process for TASAR, Rockwell Collins drafted a Project Specific Certification Plan (PSCP) and had it reviewed internally by Rockwell Collins Designated Engineering Representatives (DER) in a "dry run" of the certification process. The PSCP establishes a framework plan and schedule between the applicant and the FAA on how the applicant intends to demonstrate compliance of the new or revised system to the Federal Aviation Regulations (FARs). In the meeting with FAA approval authorities, the FAA did not see the need for a PSCP for the TASAR software, just for the installation. If the applicant already has an existing EFB installation, then the operational approval process is for a user to go directly to their Principal Operations Inspector.

Additional feedback from the FAA AIR and AFS officials included the following. TASAR will not considered by the FAA an "ADS-B IN application" but rather a performance / planning application that leverages ADS-B IN data, if available. Also, no need was identified to establish a TASAR standard. Such a standard would have ensured that different avionics vendors would produce compatible TASAR systems, but they saw no benefit in this. Their conclusion was that existing policies already cover the proposed TASAR application.

\section{E. Software Application Development}

To verify feasibility of TASAR and to facilitate its adoption for near-term operational use, NASA is developing the "Traffic Aware Planner" (TAP) software application with the intention of making it available for licensing and commercial use. The TAP software functionality and design were presented in 2013, 4 .8 Developed for NASA by Engility Corporation, TAP was designed to run on a commercial-off-the-shelf Class 2 EFB platform. TAP's flight optimization and traffic conflict management algorithms were originally developed by Engility for advanced self- 
separation research, ${ }^{7}$ and these high-performance algorithms have been extensively tested and matured for over a decade. TAP's "Auto Mode" periodically scans for time- and/or fuelsaving trajectory changes of three types: lateral-only, altitudeonly, and combination lateral/altitude changes. Using optional external data feeds such as ADS-B IN, winds, weather, and SAA activation status, TAP ensures these solutions are windoptimized and clear of known traffic and airspace conflicts. The human-machine interface (HMI), developed through an iterative human-factors design process, displays solutions textually and graphically using published fixes to facilitate voice requests to ATC. The TAP HMI includes no display of traffic or own-ship positions, thus minimizing certification issues. TAP also supports a "Manual Mode" in which the pilot enters a desired change, and the fuel/time outcomes and conflict prediction results are displayed. A sample display of the TAP application's

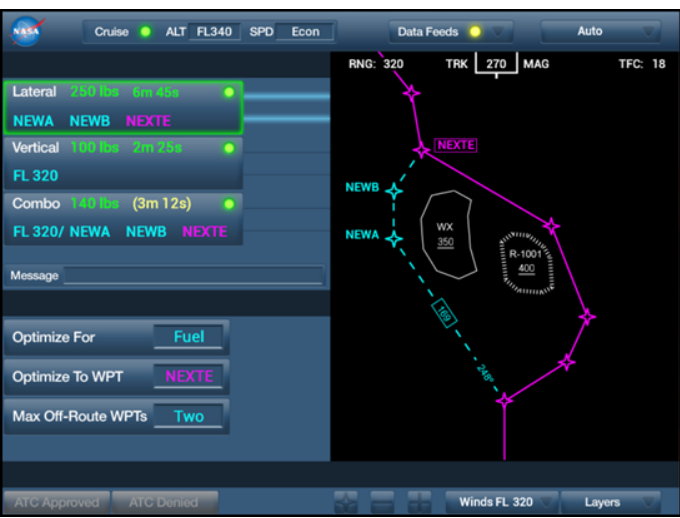

Figure 3 Updated "Auto Mode" screen of the NASA "Traffic Aware Planner" (TAP) EFB application. updated Auto Mode HMI is shown in Figure 3.

\section{F. Flight Deck Human Factors Simulations}

Two Human-in-the-Loop (HITL) simulation experiments were conducted in August 2013 and October/November 2014 in the fixed-based simulator at the University of Iowa's Operator Performance Lab (OPL) using two independent sets of 12 airline pilots as evaluators. The two experiments tested subsequent designs of the TAP HMI and associated functionality using a simulated westbound flight from JFK airport through simulated convective weather in the Midwestern states, heading in the direction of Los Angeles. TAP was installed on a tablet computer representative of a Class 2 EFB connected electronically to the simulator's avionics systems. The experiments were designed to assess human factors associated with TASAR, including effects on pilot workload, situation awareness, and distraction from flight-critical duties in non-normal situations. Acting as captain in the pilot-flying role, evaluation pilots used the TAP EFB application to manage and optimize the flight through a set of five typical TASAR use cases designed to trigger the pilot's use of TAP, while interacting with a confederate first officer, air traffic controller, and dispatcher. Physiological measures included electrocardiogram and eye-gaze tracking to objectively assess workload and area of attention. Subjective measures included the Bedford Workload Scale survey, the System Usability Score survey, the Situation Awareness Rating Technique survey, and questionnaires on specific features of the HMI. The first experiment assessed an early TAP HMI design in comparison to conducting the flight without TAP. The second experiment assessed an updated HMI design in comparison to the first experiment's results of the earlier HMI design. Both experiments included additional tasking not related to TAP to assess possible distraction caused by TAP. A photograph of the cockpit environment from the second experiment is shown in Figure 4.

Results of the two HITL experiments are currently being prepared for publication. Preliminary analysis from the first experiment indicates that there was not a significant increase in perceived cognitive workload or decrease in situation awareness when the pilots interacted with TAP compared to the baseline condition (no TAP). System Usability Scale scores indicate that TAP was perceived to be useful, understandable, intuitive, and easy to use. Additionally, the results indicate that pilot interaction with TAP did not interfere with the primary flight duties during both normal and non-normal conditions. Preliminary analysis from the second experiment suggests that the new HMI design achieved the desired improvements recommended by the pilots from the first experiment with no adverse effects on perceived cognitive workload or situation awareness. EFB task switching, i.e., using the same EFB that hosted TAP to attend to secondary tasks of generally higher criticality using other hosted applications, was successfully performed with no adverse impact on the more critical task. Finally, standalone training using a newly developed TAP Computer Based Training Module was found to be equally effective as a traditional instructor-led training briefing.

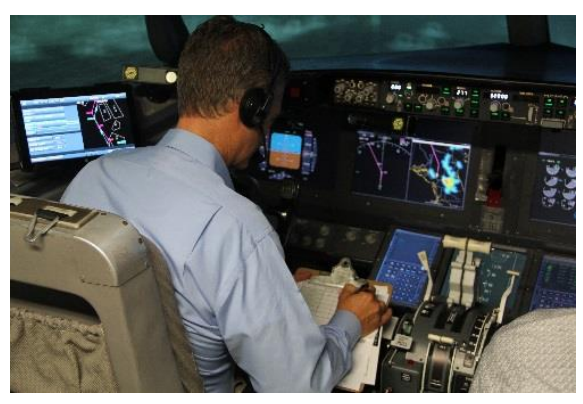

Figure 4 Operator Performance Lab simulation cab showing the TAP application to the pilot's left. Photo by M. Cover. 


\section{G. Initial Flight Trial}

An initial flight trial of the TAP software was conducted for NASA by Advanced Aerospace Solutions in November 2013 using nine experienced pilots including senior airline captains as evaluators. The Piaggio Avanti P.180 flight-test aircraft and TAP installation (using an earlier version of the TAP HMI) are shown in Figure 5. The flight trial was documented and presented in $2014 .{ }^{20}$

As of the completion of the initial flight trial, the TAP software (derived from advanced self-separation research software used previously only in a simulation laboratory) was verified to function properly in a live aircraft avionics and ADS-B IN environment. It thus successfully overcame the artifacts of real-world data characterized by dropouts, latency, and ADS-B message content fluctuations to produce viable route/altitude optimization solutions. Evaluation pilots rated TAP's usability high and workload low, and they provided valuable feedback on the HMI design that led to the design revision shown in Figure 3. Across the nine flights, twelve TASAR requests were submitted to ATC, nine were approved, and all saved fuel and flight time.
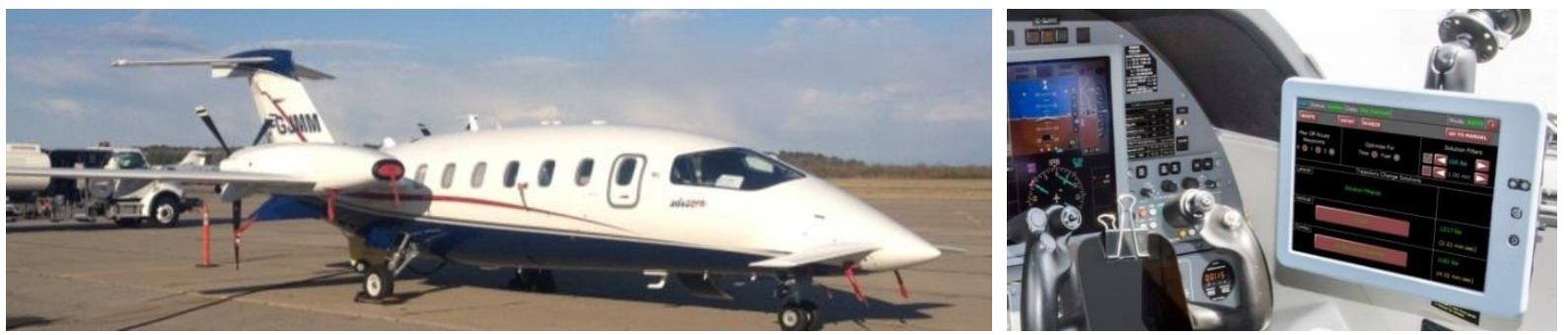

Figure 5 Piaggio Avanti flight test aircraft with TAP (and its earlier HMI) installed on a United Technologies Corporation (UTC) G500 SmartDisplay ${ }^{T M}$ EFB.

\section{Current Activities: Preparations for Airline Partner Operational Trials}

With the TASAR concept having been documented, benefits potential analyzed, FAA approval requirements determined, and the technology developed and tested in simulation and in flight, the current set of activities in the TASAR project are geared towards preparing for operational-use trials with partner airlines Alaska Airlines and Virgin America anticipated to begin in 2015. These airlines connected with NASA and TASAR in part through senior management captains from both airlines participating in the 2013 flight trial as evaluation pilots. Both airlines are recognized technology leaders and innovators, and they are engaging with NASA in a partnership to explore the operational benefits of TASAR. Alaska Airlines flies the Boeing 737NG and is equipping their aircraft with the $\mathrm{iPad}^{\mathrm{TM}}$ connected as a Class 2 EFB. Virgin America flies the Airbus 320 and is equipping their aircraft with the Astronautics Nexis ${ }^{\mathrm{TM}}$ Class 3 EFB. Taken together, these diverse platforms (aircraft and EFB) represent an excellent opportunity for NASA to develop technical integration solutions that can benefit a broad segment of the user community.

To prepare for operational-use trials by the partner airlines, NASA is pursuing several activities to advance the technology and reduce uncertainties associated with its operational use. The following subsections will describe these activities.

\section{A. Partner Airline Benefits Analyses}

To generate a baseline prediction of TASAR benefits in preparation for the partner-airline operational-use trials, an analysis was conducted to estimate annual benefits using the same modeling and simulation methodology employed for the preliminary benefits assessment ${ }^{9}$ but using the route structures, aircraft types, and flight frequencies of Alaska Airlines and Virgin America. The simulation compared historically flown trajectories without TASAR to trajectories developed with TASAR and evaluated by simulated air traffic controllers against a set of approval objectives. A total of 1,606 Alaska Airlines flights and 1,554 Virgin America flights were analyzed. For Alaska Airlines, it was estimated that between 8,000 and 12,000 gallons of fuel and 900 to 1,300 minutes could be saved annually per aircraft. For Virgin America, the estimate was about 25,000 gallons of fuel and about 2,500 minutes per aircraft of annual savings. Taking into account fuel, maintenance, and depreciation cost savings, and applying the savings fleet wide, it was estimated that two airlines could each save in excess of $\$ 5$ million per year.

Both analyses uncovered effects that illustrate the benefit of the TASAR flight-optimization capability, in particular, TAP's degrees of freedom to explore a wide range of potential routing and flight-level changes. Typically, to attempt to optimize a flight, a pilot will request a "direct" route to a downstream fix, thereby removing the assumed 
inefficiency of "dog legs" in their current route. Additionally, they will typically request a climb at some point during the flight to account for the higher operational ceiling enabled from burning fuel and reducing aircraft weight. The analyses for both airlines show that both actions by the pilot may not always produce the greatest benefits. Shown in Figure 6 are lateral trajectories from the Alaska Airlines analysis for flights between Seattle (SEA) and Newark (EWR). The flight paths influenced by TASAR requests, shown in the right plot, have a larger spread than those of the historically flown paths, shown in the left plot. Their differences reflect the advantage of using "off-route" waypoints (i.e., non-direct requests) to take advantage of non-homogeneous winds and changing atmospheric conditions. The same effect was found in the Virgin America analysis.
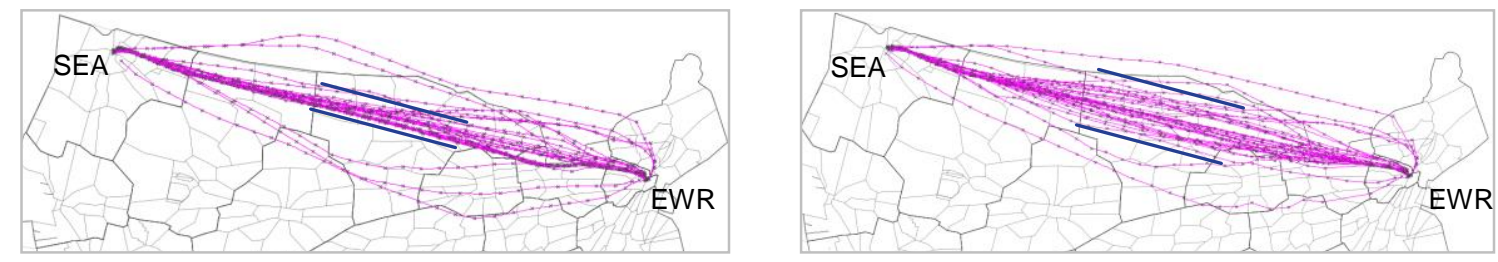

Figure 6 Trajectories between SEA and EWR showing an increased lateral spread from historically flown trajectories (left) to trajectories derived from modeling of TASAR requests (right). Data obtained from TASAR benefits analysis for Alaska Airlines.

Shown in Figure 7 are cruising altitudes from the Virgin America analysis for flights between New York (JFK) and San Francisco (SFO). Two effects are indicated from the model analysis. First, the left-hand charts shows that, on average, TASAR requests resulted in aircraft cruising at lower altitudes between JFK and SFO, particularly evident by comparing "historical" and "TASAR" percentage of time cruising at Flight Level (FL) 360 and FL 380. Second, of the five trajectory-change options shown in the right-hand chart, pure altitude changes were least often the most beneficial change. More often, combining an altitude change with a lateral change (i.e., a "combo" request) outperformed the pure altitude changes. The Alaska Airlines analysis showed similar results. These observations show that an advisory capability like TASAR aiding the aircrew in identifying the most productive change requests may yield greater user benefits as compared to the pilot using rule-of-thumb optimization.
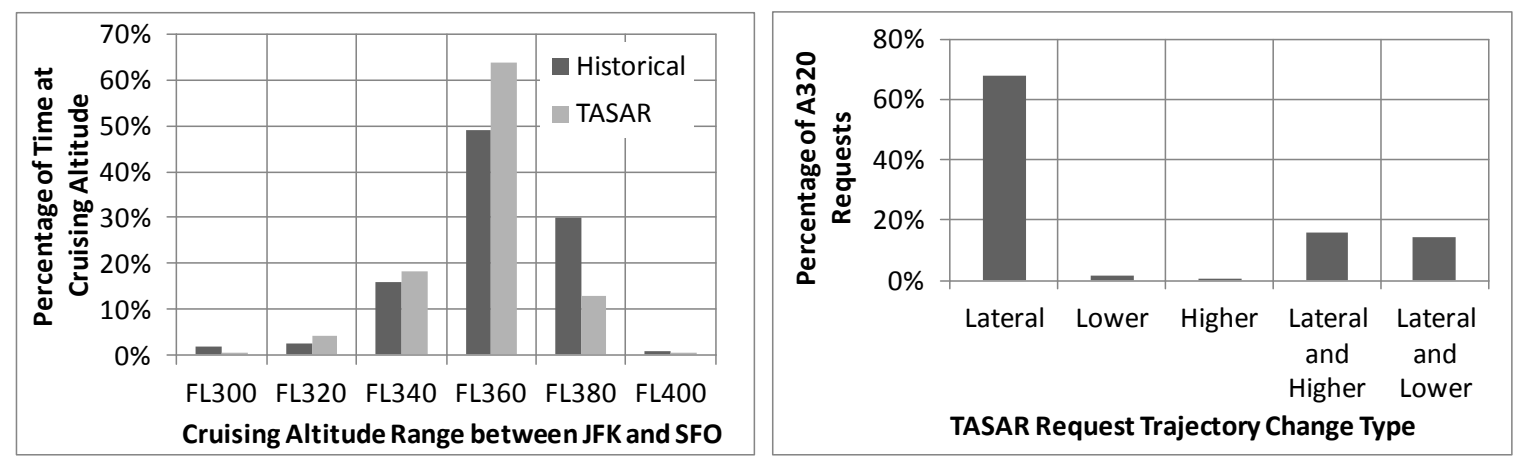

Figure 7 Altitude solutions from TASAR generally favor flying lower than historically flown trajectories and perform better when combined with lateral changes. The data was obtained from the TASAR benefits analysis for Virgin America.

\section{B. Internal Data Source Analysis}

The TAP software depends on internal data feeds from the aircraft's avionics bus, as well as an aircraft performance model, to build trajectories of the aircraft for use in flight optimization. These factors are unique for each aircraft type and avionics installation and thus require a degree of customization for installing the TAP software. To facilitate future portability to a wide variety of aircraft types, TAP is being designed to use standardized formats and interfaces wherever possible.

Some limitations have been identified, for example, in the availability of certain route data from the Flight Management Computer (FMC) of transport-category aircraft that follow the ARINC 702A-1 characteristic specification that were not limitations for the Piaggio Avanti flight-test aircraft, which follows the General Aviation Manufacturers Association (GAMA) standard. Two examples of missing information from ARINC 702A-1 are the planned cruise altitude and the waypoint names of the FMC active route. To handle these limitations in data availability, compensation mechanisms were added to the TAP software and the HMI. For the missing cruise altitude, which is used in estimating time and fuel outcomes, the HMI now provides a means for the pilot to enter and update 
this information manually. For the missing waypoint names, new functionality was added to TAP to infer the names using each waypoint's geographic coordinates and a navigation database, followed up by pilot confirmation of each waypoint name via new mechanisms added to the HMI design. The GAMA standard, as implemented in the Piaggio Avanti flight-test aircraft, does not have these limitations but has others that ARINC 702A-1 does not have. The principal example is that the GAMA FMC interface provides only eight waypoints - the most recent active-route waypoint plus the next seven waypoints - whereas the ARINC 702A-1 FMC interface will provide all future waypoints to the destination. Since TAP integrates the trajectory to the last known waypoint (preferably the destination), the HMI was modified to enable the pilot to manually enter the destination airport, thus allowing TAP to estimate the full effect of trajectory change solutions. As the adaptation analysis for Alaska Airlines and Virgin America continues, a detailed "label analysis" is being conducted to ensure every element of TAP's required dataset is identified with an appropriate source or a suitable compensation mechanism. This type of customized analysis will likely be required for every user installation, but the effort will be minimal for implementations that faithfully follow the ARINC and GAMA interface standards.

\section{External Data Sources Analysis}

In addition to internal data, TAP also leverages external data acquired through broadband internet connectivity to enhance its route optimization capability. An analysis of potential data sources, both public and commercial, was performed in preparation for the operational-use trials with the partner airlines. Categories of data types included in the analysis were convective weather, winds and temperature aloft, turbulence, icing, SAA activation status, and supplemental traffic data. Commercial sources were included in the analysis because airlines, including the two partner airlines, typically use commercial weather information service providers in their dispatch centers and have a requirement for compatibility between air and ground trajectory replanning tools.

For winds and temperature aloft, TAP is designed to use the National Oceanographic and Atmospheric Administration (NOAA) Rapid Refresh (RAP) product as its source, since it is developed specifically for aviation. RAP is an hourly update of current and forecasted winds, temperature, composite reflectivity and a number of other data products at a $13 \mathrm{~km}$ resolution with 50 vertical levels. Initially, the $40 \mathrm{~km}$ " 236 " grid has been integrated into TAP to reduce bandwidth and processing requirements. In addition to the TAP algorithms using the gridded product in its trajectory optimization search, the TAP HMI provides the pilot a visualization of the wind field at any selected flight level, thereby aiding the pilot's comprehension of TAP solutions.

For convection, TAP is designed to use polygon data to produce solutions that avoid areas of convective weather. It is assumed that operational buffers (e.g., $20 \mathrm{~nm}$ ) are included within the polygons, though a polygon buffering approach may be added to TAP's algorithms in the future. A variety of public and private sources of weather polygon data were assessed in terms of accuracy and latency. In the near term to support the upcoming operational trials with Alaska Airlines and Virgin America, TAP will be developed to incorporate the commercial data sources they already use in order to maintain consistency with data used by their dispatch centers. An additional advantage of using these commercial sources is the customization already applied to the weather products for the types of aircraft these airlines fly, as opposed to public sources which tend to be more conservative in ensuring applicability to a much wider set of aircraft types including General Aviation.

For SAAs, TAP uses a combination of an internal ARINC 424 database of defined SAA boundaries and an external connection to the FAA website "sua.faa.gov" for the latest information on airspace activation schedules. This source does not necessarily include the current status of some airspace regions such as the Warning Areas off the east coast, which can change status frequently. Other airspace data such as Temporary Flight Restrictions may also not be included in TAP's initial airspace dataset but may be added in the future.

The external data source analysis also assessed mechanisms for acquiring additional data, including turbulence, icing, and supplemental traffic data. Though these data types will not be included in TAP's initial capabilities, partially because some of these products are still maturing for aviation use, all are eventually realizable with the connectivity afforded by broadband airborne internet.

\section{TAP Software Application Enhancements}

Though the primary functionality of the TAP software was mature as of the 2013 flight trial, several enhancements are being made in preparation for the operational-use trials with the partner airlines. Some enhancements will compensate for differences in avionics data availability, as discussed above in Section B. Another enhancement is a new trajectory integrator being developed specifically for the TAP application. The new integrator will put fidelity where it is needed (e.g., modeling cruise altitude changes correctly) and remove it where it is not (e.g., detailed descent modeling), resulting in a more flexible and accurate system tuned for TAP's primary use in optimizing the en-route phase of flight. Detailed modeling in the descent (which adds significantly to the complexity of the trajectory 
integrator) is not critical to the accuracy of TAP's outcome computations because the descent will typically be unchanged by TAP and therefore time/fuel estimates will not be significantly affected by how accurately the descent is modeled.

The most visible enhancement to the TAP software is the near-total redesign of the HMI from the versions tested in the 2013 HITL experiment and flight trial. Using evaluation-pilot data from these activities, human factors principles were applied throughout all of the HMI's features to enhance usability and situation awareness without significantly affecting pilot workload. In particular, three primary screens were consolidated to two screens, with route visualization now appearing as a data-enriched moving map directly in both the Auto Mode (shown in Figure 3) and Manual Mode screens. The Auto Mode screen now provides a clear indication of the best overall optimization solution, and a preview feature enables the pilot to quickly review all three solutions graphically. The Manual Mode screen provides a more user-friendly interface for specifying a custom route, including tapping directly on the map to trigger TAP to auto-select the nearest named waypoint to the selected location. Color-coding is used to indicate conflicted route segments, and a message window provides supplemental information on any conflicts. The updated HMI also now includes a Startup Checklist screen for entering the compensation data discussed earlier in Section B and a Performance/Constraints screen for updating this information and adding optional flight-specific performance values and trajectory constraints to enhance TAP's performance calculations. In addition, styling was updated throughout the HMI to be more consistent with the design of modern tablet applications.

Complementary to the HMI redesign is an architectural change to enable the TAP HMI and TAP computing engine to run on separate processors, which broadens the variety of EFB devices and systems that can support the TAP software. For instance, the $\mathrm{iPad}^{\mathrm{TM}}$ will be able to host the TAP HMI, while the TAP engine can take advantage of a more powerful onboard processor. The new architecture will also support application task-switching on the EFB, whereby the TAP HMI can go dormant while the pilot uses other applications; meanwhile, the TAP engine continues its processing and quickly reestablishes communication with the HMI when available.

As part of the HMI redesign process, the HMI was prototyped using the Crank Storyboard ${ }^{\mathrm{TM}}$ Suite to provide an interactive mockup of the HMI. To assist the partner airlines in training their pilots on the TAP software, the mockup was further developed into a Computer-Based Training (CBT) module. The CBT enhances learning by providing the trainee an opportunity to interact with the HMI by touching buttons and seeing the resulting effect. The CBT was evaluated against traditional instructor-led training during the second HITL simulation experiment and found to be effective as a stand-alone training tool. NASA intends to provide the CBT to users of the TAP software to help accelerate user adoption of TASAR.

\section{E. Flight Trial \#2}

A second flight trial in the Piaggio Avanti is being conducted in the late spring of 2015. The goal of the flight trial is to increase operational readiness in preparation for the operational-use trials with the partner airlines. Specific objectives include: (1) verifying TAP operates correctly on the EFB and associated hardware intended by the partner airlines; (2) verifying the ability in flight to retrieve and incorporate additional external data via internet into TAP processing; (3) assessing a methodology to quantify the accuracy and stability of TAP computed outcomes (e.g., time and fuel saved); (4) determining the acceptability to controllers and pilots of various TASAR request characteristics; (5) assessing usability of the updated TAP HMI in flight; and (6) assessing the application of crew resource management practices to TASAR operations. Six round-trip flights are planned in Washington, Jacksonville (ZJX), and Atlanta (ZTL) Center airspace. Observers will be stationed in both the aircraft and the ZJX and ZTL Air Route Traffic Control Centers to gather information from both sides of the operation. Two evaluation pilots per flight will be aboard the Piaggio Avanti, one from each of Alaska Airlines and Virgin America. TAP will be installed on two iPad Airs ${ }^{\mathrm{TM}}$, one in the cockpit and one in the cabin, for their use and assessment. An airborne internet connection will provide real-time connectivity to NOAA for winds and temperature data, the FAA SAA site for SAA activation status, and to a WSI server for convective weather data. Upon completion of this second flight trial, the TASAR technology and procedures are expected to have reached sufficient maturity to proceed with operational-use testing in partnership with Alaska Airlines and Virgin America in revenue flight.

\section{Future Activities and Direction: Operational Trials, Integration, \& Growth}

Though not finalized as of the submission of this paper, the plans for conducting operational trials of TASAR with Alaska Airlines and Virgin America are progressing, with first flights expected to occur in late 2015 or early 2016. NASA is defining an aircraft adaptation process that will extend the experience gained from adapting TAP to the Piaggio Avanti flight-test aircraft to the customized adaptations required for the two airlines. The process includes designing and documenting the EFB hardware/software environment for hosting TAP, conducting a detailed data- 
input analysis of internal avionics data based on the ARINC 834 specification (i.e., the EFB interface standard), conducting a similar analysis for non-834 data including internet sources and the ARINC 424 navigation database, identifying the source and format of aircraft performance data for TAP's trajectory integrator, conducting bench tests of TAP running on the airlines' designated EFB hardware to assess mutual performance effects, and defining the processes to be used for software deployment to the aircraft and eventual data collection. NASA will also provide assistance to the airlines in their application to the FAA for operational approval by providing artifacts from its research and analyses. Exercising these processes for such disparate aircraft platforms as the Piaggio Avanti, a Boeing $737 \mathrm{NG}$, and an Airbus A320, and EFB platforms of the UTC SmartDisplay ${ }^{\mathrm{TM}}$, the iPad $^{\mathrm{TM}}$, and the Astronautics Nexis $^{\mathrm{TM}}$, will enable NASA to define a more generalized process for industry use in further commercialized deployments. Once the airlines are conducting TASAR operations, NASA will conduct joint analysis with the airlines of the realized benefits and any operational issues. This will begin a new phase of increasing the maturity of the TAP software through its operational use and identifying with the airlines any additional capabilities and functions to increase operational acceptability and performance.

One primary focus for further TASAR development is expected to be some form of integration between the flight deck and the dispatcher's station. In airline operations, the captain and dispatcher share joint responsibility for the conduct of the flight, and flight-path changes of a significant degree (typically those exceeding $100 \mathrm{~nm}$ lateral deviation or $4000 \mathrm{ft}$ vertical change) generally require concurrence of both parties prior to execution. Whether the proposed trajectory change originates from the dispatcher or supporting ground-based flight-optimization tools, for example NASA's Dynamic Weather Routes, ${ }^{21}$ or from flight-deck tools such as TAP, the need for some level of airground information sharing is evident to enable the most effective overall process for achieving optimized trajectories. NASA's Airspace Technology Demonstration Project will be exploring such concepts and mechanisms for integration.

In addition to dispatch integration, other areas to be explored include data source expansion, optimization enhancements, operational constraints analysis and handling, and possible future applications involving data communications integration with ATC. These investigations will be conducted in partnership with airlines and industry to the greatest extent possible.

\section{Summary}

NASA has been developing and testing the Traffic Aware Strategic Aircrew Requests (TASAR) concept for aircraft operations featuring a NASA-developed cockpit automation tool, the Traffic Aware Planner (TAP), which computes traffic/hazard-compatible route changes to improve flight efficiency. The TAP technology is anticipated to save fuel and flight time and thereby provide immediate and pervasive benefits to the aircraft operator, as well as improving flight schedule compliance, passenger comfort, and pilot and controller workload. This paper has summarized the progress made in developing TASAR from an operational concept to near readiness for operationaluse trials by two airlines intending to work in partnership with NASA, Alaska Airlines and Virgin America.

Preliminary benefits analysis and subsequent airline-specific benefits analyses have shown the potential for significant user benefits in fuel-burn and flight-time savings. An analysis of operational hazards and safety indicates TASAR would likely receive a Failure Effects Classification no greater than "Minor Effect." An analysis of regulatory compliance requirements and subsequent vetting with FAA officials has confirmed TASAR's qualification as a Type B software application that requires only Principal Operations Inspector approval for operational use. The TAP software itself has undergone a human-factors assessment in two simulation experiments and its functionality vetted in an initial flight trial, with a second flight trial scheduled for June 2015. Upcoming work will focus on final preparations for initial deployment on Alaska Airlines and Virgin America aircraft for operational-use testing.

TASAR represents a seminal advancement of the aircraft's role in airspace operations, promoting it from just a passive recipient of ATC instructions to an active trajectory manager in the dynamic airspace. The concept integrates and leverages three elements critical to the advancement of future autonomy in aircraft operations: advanced trajectory-planning automation, the emerging NextGen air-to-air traffic surveillance system, and networked connectivity to an array of internet-based information sources. NASA's Aeronautics Research Mission Directorate is strategically focused on promoting operational autonomy in the National Airspace System, and TASAR is expected to be a principal catalyst towards this transformation by leveraging a unique combination of key attributes that support low-risk, near-term deployment by the user community. 


\section{References}

1 Ballin, M.G. and Wing, D.J., "Traffic Aware Strategic Aircrew Requests (TASAR)," AIAA-2012-5623, AIAA Aviation Technology, Integration, and Operations Conference (Indianapolis, IN, 2012), AIAA, Washington, DC, 2012.

2 Henderson, J., "Traffic Aware Strategic Aircrew Requests (TASAR) Concept of Operations", NASA/CR-2013-218001, NASA Langley Research Center, Hampton, VA, 2013.

3 Ballin, M.G. and Wing, D.J., "Method and Apparatus for Generating Flight-Optimizing Trajectories," U.S. Patent 8,977,482, March 10, 2015.

4 Woods, S., Vivona, R., Roscoe, D., Lefebvre, B., Wing, D., and Ballin, M., "A Cockpit-based Application for Traffic Aware Trajectory Optimization," AIAA-2013-4967, AIAA Guidance, Navigation and Control Conference (Boston, MA, 2013), AIAA, Washington, DC, 2013.

5 NASA Strategic Plan 2014, http://www.nasa.gov/sites/default/files/files/2014_NASA_Strategic_Plan.pdf

6 Wing, D.J. and Cotton, W.B., "For Spacious Skies: Self-Separation with 'Autonomous Flight Rules' in US Domestic Airspace”, AIAA-2011-6865, AIAA Aviation Technology, Integration, and Operations Conference (Virginia Beach, VA, 2011), AIAA, Washington, DC, 2011.

7 Karr, D.A., Vivona, R.A., Roscoe, D.A., and Wing, D.J., “Autonomous Operations Planner: A Flexible Platform for Research in Flight-Deck Support for Airborne Self-Separation", AIAA-2012-5417, AIAA Aviation Technology, Integration, and Operations Conference (Indianapolis, IN, 2012), AIAA, Washington, DC, 2012.

8 Wing, D., Ballin, M., Koczo, S., Vivona, R., and Henderson, J. "Developing an Onboard Traffic-Aware Flight Optimization Capability for Near-Term Low-Cost Implementation," AIAA-2013-4231, AIAA Aviation Technology, Integration, and Operations Conference (Los Angeles, CA, 2013), AIAA, Washington, DC, 2013.

9 Henderson, J. and Idris, H., Preliminary Benefits Assessment of Traffic Aware Strategic Aircrew Requests (TASAR)," AIAA-2012-5684, AIAA Aviation Technology, Integration, and Operations Conference (Indianapolis, IN, 2012), AIAA, Washington, DC, 2012.

${ }^{10}$ Bilimoria, K. D., Sridhar, B., Chatterji, G., Sheth, K. S., and Grabbe, S., "FACET: Future ATM Concepts Evaluation Tool," Air Traffic Control Quarterly, Vol. 9, No. 1, 2001, pp. 1-20.

${ }^{11}$ Koczo, S., "Analysis of Operational Hazards and Safety Requirements for Traffic Aware Strategic Aircrew Requests (TASAR)," NASA/CR-2013-218002, NASA Langley Research Center, Hampton, VA, 2013.

${ }^{12}$ Koczo, S., \& Wing, D., "An Operational Safety and Certification Assessment of a TASAR EFB Application”, Digital Avionics Systems Conference (East Syracuse, NY, 2013), 2A1-1-2A1-18. doi:10.1109/DASC.2013.6712530, 2013.

${ }^{13}$ Kozco, S., "TASAR Certification and Operational Approval Requirements - Analyses and Results", submitted for publication as a NASA Contractor Report.

${ }^{14}$ SAE ARP 4761, Guidelines and Methods for Conducting the Safety Assessment Process on Civil Airborne Systems and Equipment.

${ }^{15}$ AC 23.1309-1, System Safety Analysis and Assessment for Part 23 Airplanes, Federal Aviation Administration, Washington, DC.

${ }^{16}$ AC 25.1309-1, System Design and Analysis, Federal Aviation Administration, Washington, DC.

${ }^{17}$ RTCA DO-264 / EUROCAE ED-78A, “Guidelines for Approval of the Provision and Use of Air Traffic Services supported by Data Communications".

${ }^{18}$ AC 120-76B, "Guidelines for the Certification, Airworthiness, and Operational Approval of Electronic Flight Bag Computing Devices", Federal Aviation Administration, Washington, DC.

${ }^{19}$ AC 20-115, RTCA, Inc., Document RTCA/DO-178B, Federal Aviation Administration, Washington, DC.

${ }^{20}$ Maris, J., Haynes, M., Wing, D., Burke, K., Henderson, J., and Woods, S. "Traffic Aware Planner (TAP) Flight Evaluation," AIAA-2014-2166, AIAA Aviation Technology, Integration, and Operations Conference (Atlanta, GA, 2014), AIAA, Washington, DC, 2014.

${ }^{21}$ McNally, D., Sheth, K., Gong, C., Love, J., Lee, C., Cheng, J., and Sahlman, S. "Dynamic Weather Routes: A Weather Avoidance Concept for Near-Term Trajectory-Based Operations," accepted for publication at the International Council on Aeronautical Sciences, Brisbane, Australia, Sep. 2012. 